\title{
Sixth mode in massive gravity
}

\author{
Marco Celoria, ${ }^{1, *}$ Denis Comelli, ${ }^{2, \dagger}$ and Luigi Pilo ${ }^{3,4, \$}$ \\ ${ }^{1}$ Gran Sasso Science Institute (INFN), Via Francesco Crispi 7, I-67100 L'Aquila, Italy \\ ${ }^{2}$ INFN, Sezione di Ferrara, I-44122 Ferrara, Italy \\ ${ }^{3}$ Dipartimento di Scienze Fisiche e Chimiche, Università dell'Aquila, I-67010 L'Aquila, Italy \\ ${ }^{4}$ INFN, Laboratori Nazionali del Gran Sasso, I-67010 Assergi, Italy
}

(Received 21 March 2018; published 12 September 2018)

\begin{abstract}
Generic massive gravity models in the unitary gauge correspond to a self-gravitating medium with six degrees of freedom (d.o.f.). It is widely believed that massive gravity models with six d.o.f. have an unavoidable ghostlike instability; however, the corresponding medium has stable phononlike excitations. The apparent contradiction is solved by the presence of a nonvanishing background pressure and energy density of the medium that opens up a stability window. The result is confirmed by looking at linear stability on an expanding Universe, recovering the flat space stability conditions in the small wavelength limit. Moreover, one can show that under rather mild conditions, no ghostlike instability is present for any wavelength. As a result, exploiting the medium interpretation, a generic massive gravity model with six d.o.f. is perfectly viable.
\end{abstract}

DOI: 10.1103/PhysRevD.98.064016

\section{INTRODUCTION}

In recent years there has been a renewed interest in massive gravity, see, for instance, the reviews [1-3]. One of the main issues of massive gravity is that a randomly picked model on Minkowski space propagates six degrees of freedom (d.o.f.) and among the two scalar modes one leads to ghost instabilities $[4,5,1-3]$. This is the case irrespectively if the proposed theories are Lorentz invariant [4] or Lorentz breaking [6,5].

A lot of effort has been devoted in finding suitable models in which only five healthy d.o.f. are present. At the nonperturbative level, when a residual Lorentz symmetry is imposed in the massive gravity action, only the de RhamGabadadze-Tolley (dRGT) model [7] has such a feature; if only rotational symmetry is imposed many choices are available $[6,8,9]$. Unfortunately, in both cases the special choice of the ghost free action is not protected by symmetries. In the Lorentz invariant case there are a number of additional phenomenological difficulties: the absence of a spatially flat Friedmann-Lemaitre-Robertson-Walker (FLRW) cosmological solution, the presence of the van Dam-VeltmanZakharov (vDVZ) discontinuity [10-12], and very tight constraints on the model parameters from unitarity [13].

\footnotetext{
*marco.celoria@gssi.infn.it

comelli@fe.infn.it

†uigi.pilo@aquila.infn.it
}

Published by the American Physical Society under the terms of the Creative Commons Attribution 4.0 International license. Further distribution of this work must maintain attribution to the author(s) and the published article's title, journal citation, and DOI. Funded by SCOAP .
In general, the recent detection of gravitational waves $[14,15]$ poses strong constraints on modified gravity theories relevant for dark energy; see, for instance, Refs. [16-19] disfavoring theories in which gravitational propagation velocity is modified.

In this paper we reconsider the faith of the sixth mode in light of the interpretation of massive gravity as a selfgravitating medium [20-22]. The crucial point is that the energy density and pressure of the medium when present can open up a region of stability for massive gravity models with healthy six d.o.f. The very same mechanism guaranties that two scalar phononlike modes of perfect fluid are stable; the argument can be extended to a general medium.

Technically, we stick to the following path:

(i) A generic massive gravity model with six d.o.f. around Minkowski space is intrinsically plagued by a scalar ghost.

(ii) In the medium interpretation of the massive gravity models we find that in the absence of dynamical gravity, the phononlike excitations of such models are stable.

(iii) For consistency, the presence of the medium requires a nonzero energy density $\rho$ and pressure $p$, which, when gravity is switched on, leads to the departure from flat space as a consistent background.

(iv) Analyzing perturbations around a FLRW background in the short wavelength limit, the same stability conditions for a medium on Minkowski spacetime are recovered.

This shows that massive gravity models with six d.o.f. can be intrinsically stable. 
In a previous work [21], done by some of us, we missed the above fundamental point. We were aware that in general six d.o.f. were present, but at that time we were too concentrated in setting the general formalism matching massive gravity (MG) and the media formalism. We did not discuss stability issues so we did not get the point on the sixth mode. In fact, we considered only special cases where only five d.o.f. were present.

The outline of the paper is as follows. In Sec. II we study the stability of a generic medium in Minkowski space. In Sec. III we relate massive gravity with a self-gravitating medium by exploiting the unitary gauge. Section IV is devoted to the analysis of stability for a self-gravitating medium around a FLRW background. The various possible phases of massive gravity are discussed in Sec. V. Our conclusions are given in Sec. VI.

\section{A MEDIUM IN MINKOWSKI SPACE}

Let us start from a scalar field theory of the form

$$
S_{m}=\int d^{4} x \sqrt{-g} U(\partial \varphi),
$$

where $U$ is the Lagrangian for four derivatively coupled scalar fields $\varphi^{A}, A=0,1,2,3$ invariant under shift symmetry $\varphi^{A} \rightarrow \varphi^{A}+c^{A}$ with $\partial_{\mu} c^{A}=0$. As discussed in detail in Refs. [20-24] such an action can be used to give an effective description of the dynamics of selfgravitating media coupled with gravity. The low energy excitations (phonons) can be described by suitable fluctuations of the $\varphi^{A}$; see Appendix A for details. In this chapter we consider the perturbations $\pi^{A}$ around flat space

$$
\varphi^{A}=\bar{\varphi}^{A}+\pi^{A} .
$$

It is easy to see that in order to have a energy momentum tensor (EMT) constant, the background value of the scalar fields is $\bar{\varphi}^{A}=x^{A}$. The presence of the fluid breaks spontaneously the Lorentz group. Notice that setting $\bar{\varphi}^{A}=$ 0 it would imply that there is no background fluid $(\rho=p=0)$. The Lagrangian $U$ gives the following background EMT:

$T_{\text {Mink }}^{\mu \nu}=\left(\rho \delta_{0}^{\mu} \delta_{0}^{\nu}+p \delta_{i}^{\mu} \delta_{i j} \delta_{j}^{\nu}\right)=p \eta^{\mu \nu}+(\rho+p) u^{\mu} u^{\nu}$,

where $\rho$ and $p$ are constants that give the energy density and the pressure evaluated on flat space by Eq. (A6), measured by observers with 4 -velocity $u^{\mu}=(1, \overrightarrow{0})$.

The first thing to notice is that if one requires the background EMT to be Lorentz invariant ${ }^{1}$ then we have to impose $\rho+p=0$, i.e., $T_{\text {Mink }}^{\mu \nu}=p \eta^{\mu \nu}$, i.e., the fluid has

\footnotetext{
${ }^{1}$ Of course this statement is based on symmetry and not on the equations of motion.
}

the same equation of state of a cosmological constant. From the media-fluid interpretation it is rather natural that the vacuum state breaks Lorentz symmetry by its presence; that is precisely what happens in any solid state lab. In what follows we will consider the case $\rho+p \neq 0$, while the exotic case $\rho+p=0$ will be studied elsewhere.

Decomposing the $\pi^{A}$ fields according to the $S O(3)$ vector and scalar perturbations: $\varphi^{0}=t+\pi_{0}$ and $\varphi^{i}=x^{i}+$ $\partial_{i} \pi_{l}+V^{i}$ (with $\partial_{i} V^{i}=0$ ), we get that for the scalar modes the quadratic Lagrangian is ${ }^{2}$

$$
\begin{aligned}
L_{\pi}^{(s)}= & \frac{k^{2}}{2}\left(\hat{M}_{1}+p+\rho\right) \pi_{l}^{\prime 2}+\hat{M}_{0} \pi_{0}^{\prime 2}+\frac{k^{2}}{2} \hat{M}_{1} \pi_{0}^{2} \\
& +k^{2}\left(\hat{M}_{1}-2 \hat{M}_{4}\right) \pi_{0}^{\prime} \pi_{l}+k^{4}\left(\hat{M}_{3}-\hat{M}_{2}\right) \pi_{l}^{2},
\end{aligned}
$$

where we have introduced the mass parameters $M_{i}$, defined in terms of the derivatives of the medium Lagrangian (2.1); their explicitly expression (A7) is given in Appendix A. Such parameters are quite useful in order to classify the various media with respect to their mechanical and thermodynamical properties. ${ }^{3}$ Moreover, it is convenient to set $\hat{M}_{a}=M_{\mathrm{Pl}}^{2} M_{j}, j=0,1,2,3,4$. The linear expansion of the medium action gives

$$
S_{\pi}^{(1)}=\int d^{4} x\left[\sigma \dot{\pi}^{0}+(\sigma-p-\rho) \partial_{i} \pi^{i}\right]=0
$$

where $\sigma$ is the background entropy per particle. By integration by parts, the linear action is zero upon the constancy in space and in time of $\sigma$. Thus no condition on $p$ and $\rho$ needs to be imposed in sharp contrast with the case where dynamical gravity is present, as we shall see later.

For transverse vectors $\vec{V}=\left(V^{1}, V^{2}, V^{3}\right)$ one gets

$$
L_{\pi}^{(V)}=\frac{1}{2}\left(\hat{M}_{1}+p+\rho\right) \vec{V}^{\prime 2}-k^{2} \hat{M}_{2} \vec{V}^{2} .
$$

We consider the case of generic $M_{i}$ values, thus no tuning and/or additional symmetries are imposed. From the kinetic terms in Eq. (2.4) it is clear that there are two critical points: $\hat{M}_{1}+p+\rho=0$ and $\hat{M}_{0}=0$, where at least one d.o.f. can be integrated and the stability analysis has to be redone. As soon as we are away from such points we can study the stability looking to the total energy, or, equivalently, the Hamiltonian (see Appendix B). In the scalar sector the total energy is given by

\footnotetext{
${ }^{2}$ We can get such a Lagrangian from Eq. (B10) taking only the 3rd and 4th diagonal entries and then imposing the Minkowski space limit $\left(\phi^{\prime}=a=1, \mathcal{H}=0\right)$.

${ }^{3}$ From the mechanical point of view we have perfect fluids with $M_{1,2}=0$, solids $M_{1}=0$, superfluids $M_{2}=0$. and supersolids $M_{1,2} \neq 0$. From the thermodynamical point of view we have adiabatic media when $M_{1}=0$, isentropic media when $M_{0}=0$, isentropic perfect fluids when $\hat{M}_{1}+\rho+p=0$.
} 


$$
\begin{aligned}
E_{s}= & \hat{M}_{0} \pi_{0}^{\prime 2}+\frac{k^{2}}{2}\left(\hat{M}_{1}+p+\rho\right) \pi_{L}^{\prime 2}+k^{4}\left(\hat{M}_{2}-\hat{M}_{3}\right) \pi_{L}^{2} \\
& -\frac{k^{2}}{2} \hat{M}_{1} \pi_{0}^{2} .
\end{aligned}
$$

Imposing that the energy is bounded from below in both the scalar and vector sectors leads to (see Appendix B)

$$
\begin{array}{ll}
M_{0}>0, & -(p+\rho)<\hat{M}_{1}<0, \\
M_{2}>0, & M_{2}>M_{3} .
\end{array}
$$

Clearly, when $\rho+p>0$, stability is possible, as it should be. Actually, the conditions of dynamical and thermodynamical stability for a perfect fluid are the same [25]. It is interesting to note that the previous stability conditions do not hold in the Lorentz invariant case. ${ }^{4}$ Indeed, requiring that $T_{\text {Mink }}^{\mu \nu}$ be proportional to $\eta^{\mu \nu}$ implies that $p+\rho=0$; thus only the barotropic equation of state $w=-1$ is viable and the stability windows closes down. ${ }^{5}$

Though Stuickelberg fields are perfectly suitable for studying stability, they are not invariant under the shift symmetries of the action and their physical interpretation is not direct. It is convenient to introduce [25,22] as observables the energy density perturbation $\delta \rho$, the pressure perturbation $\delta p$, and the entropy per particle perturbation $\delta \sigma$ given by

$\delta \sigma=2\left(\hat{M}_{0} \pi_{0}^{\prime}-\hat{M}_{4} k^{2} \pi_{l}\right), \quad \delta \rho=\delta \sigma-(p+\rho) k^{2} \pi_{l}$,

$\delta p=c_{s}^{2} \delta \rho+\left(c_{b}^{2}-c_{s}^{2}\right) \delta \sigma$,

where

$c_{b}^{2}=-\frac{M_{0}}{M_{4}}, \quad c_{s}^{2}=\frac{6 \hat{M}_{4}^{2}+2 \hat{M}_{0}\left(\hat{M}_{2}-3 \hat{M}_{3}\right)}{3 \hat{M}_{0}(p+\rho)}$.

The equations of motion derived from (2.4) can be written as

$$
\begin{aligned}
\delta \sigma^{\prime \prime}+c_{\sigma}^{2} k^{2} \delta \sigma+\frac{\left(c_{\rho}^{2}-c_{b}^{2}\right) \hat{M}_{1}}{\left(\hat{M}_{1}+p+\rho\right)} k^{2} \delta \rho & =0, \\
\delta \rho^{\prime \prime}+k^{2} c_{\rho}^{2} \delta \rho+k^{2}\left(c_{b}^{2}-c_{\rho}^{2}\right) \delta \sigma & =0,
\end{aligned}
$$

where $c_{\rho}^{2}$ and $c_{\sigma}^{2}$ depend on $\left\{M_{a}\right\}$. The evolution equation (2.11) has been derived in the hypothesis that $M_{1} \neq 0$. If $M_{1}=0$ we simply have $\sigma^{\prime}=0$. It is clear that the second scalar mode, beside $\delta \rho$, is the entropy per particle $\delta \sigma$; from the equations of motion both are linearly stable when Eq. (2.8) hold.

\footnotetext{
${ }^{4}$ Here we do not consider that the case where $\bar{T}^{\mu \nu}$ is not Lorentz invariant but the quadratic action Lagrangian is Lorentz invariant.

${ }^{5}$ Unless we require $M_{0}=0$ or $\hat{M}_{1}+\rho+p=0$ resulting in less than six propagating d.o.f.
}

\section{SELF-GRAVITATING MEDIUM AND MASSIVE GRAVITY}

Let us now include dynamical gravity in our picture. The medium becomes self-gravitating by simply coupling minimally the scalar fields with gravity. Namely, one consider the following action

$$
S=M_{\mathrm{Pl}}^{2} \int d^{4} x \sqrt{-g} R+\int d^{4} x \sqrt{-g} U\left(\partial \varphi, g_{\mu \nu}\right),
$$

where $R$ is the Ricci scalar; as before, $U$ is the medium Lagrangian containing four derivatively coupled scalar fields minimally coupled with gravity (see Appendix A for more details). Perturbations around flat space are studied by taking

$$
d s^{2}=\eta_{\mu \nu}+h_{\mu \nu} d x^{\mu} d x^{\nu}, \quad \varphi^{A}=\bar{\varphi}^{A}+\pi^{A} .
$$

By definition, in the unitary gauge the scalar fields fluctuations are gauged away and their value coincides with the background values $\varphi_{(U)}^{A}=x^{\mu} \delta_{\mu}^{A}$; all perturbations are in the metric and we set $h_{\mu \nu}=h_{\mu \nu}^{(U)}$. In flat space, the expansion of the action (2.1) generates a linear term

$$
\mathcal{L}_{1}^{(U)} \equiv \frac{1}{2} T_{\text {Mink }}^{\mu \nu} h_{\mu \nu}^{(U)}
$$

proportional to the EMT of the medium $T_{\text {Mink }}^{\mu \nu}$ (2.3). At the quadratic level there is a contribution coming from both the Einstein-Hilbert Lagrangian $\mathcal{L}_{g}^{(U)}$ and the medium action; the later can be parametrized in the form $[6,5,1,26]$

$$
\begin{aligned}
\mathcal{L}_{m}^{(U)}= & \left.\sqrt{-g} U\right|_{\mathcal{O}(2)} \\
= & \frac{M_{\mathrm{Pl}}^{2}}{4}\left[\lambda_{0}^{2} h_{00}^{(U) 2}+2 \lambda_{1}^{2} h_{0 i}^{(U) 2}-2 \lambda_{4}^{2} h_{00}^{(U)} h_{i i}^{(U)}\right. \\
& \left.+\lambda_{3}^{2} h_{i i}^{(U) 2}-\lambda_{2}^{2} h_{i j}^{(U) 2}\right],
\end{aligned}
$$

whose structure is the origin of the name massive gravity theories. Thus, in the unitary gauge a self-gravitating medium is equivalent to a general massive gravity theory. The parameters $\lambda_{i}$ are given in terms of suitable first and second derivatives of the function $U$ computed on Minkowski space background and their expression can be derived from the parameters $M_{i}$ used to study the dynamics of a medium $[21,22]$ by

$$
\begin{aligned}
M_{0} & =\lambda_{0}^{2}-\frac{\rho}{2 M_{\mathrm{Pl}}^{2}}, \quad M_{1,2}=\lambda_{1,2}^{2}-\frac{p}{M_{\mathrm{Pl}}^{2}}, \\
M_{3,4} & =\lambda_{3,4}^{2}-\frac{p}{2 M_{\mathrm{Pl}}^{2}},
\end{aligned}
$$

and given in Eq. (A7). To be as general as possible we imposed on the mass terms only rotational invariance. Such 
a choice is rather natural in the medium picture the spontaneous breaking of Lorentz symmetry originates simply by the presence of the medium. Strictly speaking only the presence of the term $\lambda_{2}^{2}$ corresponds to a mass term for the spin two component of the graviton; however, we will generically refer to massive gravity when at least one of the $\lambda_{i}^{2}$ is nonvanishing. Insisting on having a Lorentz symmetric background configuration constrains the value the $\lambda_{a}$ and the quadratic expansion (3.4) reduces to

$$
\mathcal{L}_{\text {mass }}^{(2)}=\frac{M_{\mathrm{Pl}}^{2}}{4}\left[A h_{\mu \nu}^{(U)} h_{\alpha \beta}^{(U)} \eta^{\mu \alpha} \eta^{\nu \beta}+B\left(h_{\mu \nu}^{(U)} \eta^{\mu \nu}\right)^{2}\right] ;
$$

thus $\lambda_{0}^{2}=A+B, \quad \lambda_{1}^{2}=\lambda_{2}^{2}=-A$ and $\lambda_{3}^{2}=\lambda_{4}^{2}=B$. Of course, in this case the corresponding medium is rather special; Lorentz invariance forces having $p=-\rho$ and thus $T_{\text {Mink }}^{\mu \nu}=-\rho \eta^{\mu \nu}$.

Before proceeding let us point out that a consistent study of gravity perturbations around flat space conflicts with the fluid-medium picture. Take, for instance, the unitary gauge; flat space is a solution of the background equations only if $\rho=p=0$, as a result no fluid is present.

Physically it simply means that when gravity matters, the consistent background is not flat: the presence of a nonvanishing $p$ and $\rho$ wants to warp the background. To study perturbations in a consistent way, keeping $p$ and $\rho$ nonvanishing, one has to look carefully at the scale involved.

(1) If the curvature radius is large compared to the typical wavelength of the perturbations we are considering, the flat space picture is adequate and the fluctuations of the spacetime metric can be neglected.

(2) The curvature radius is comparable to the typical wavelength of the perturbations; as a result, the background solution has to be amended and the metric fluctuations are important.

In case 1, at very large momentum and energy, curvature is negligible together with the mixing of the Stückelberg fields with the metric, much like in a spontaneously broken gauge theory where the ultraviolet behavior is captured by $\pi$ 's alone. Thus, one can forget about gravity and simply study the effective quadratic Lagrangian for the $\pi$ 's obtained by expanding $U$ and the stability analysis is the one given in the previous section. When case 2 applies we need to consider a consistent background, the natural one is an expanding FLRW Universe that will be discussed in the next section.

One may be tempted to simply forget about the restriction imposed by the background equations, however, this is not an option and it leads to a contradiction. Indeed, as shown in Appendix B, the quadratic Lagrangian in unitary gauge does not depend on $p$ and $\rho$ and for generic values of the masses two d.o.f. are present and one is a ghost. On the other hand, employing the Newtonian gauge, the quadratic action depends on both $p$ and $\rho$ but this time, though the kinetic matrix for the relevant fields is not positive definite, three d.o.f. seem to be present. As expected, to reconcile the two gauges one has that $\rho=p=0$. The bottom line is that, a self-gravitating medium cannot be consistently studied in flat space unless there is no medium. We stress again that the medium picture is not available when Lorentz invariance is imposed on the background EMT, then $p+\rho=0$. In this case, the stability windows close down, making clear, once again, the key role played by the background pressure and energy density of the medium, which have to be different from zero to avoid instabilities in the presence of six d.o.f..

\section{STABILITY IN AN EXPANDING UNIVERSE}

Let now consider the case when the perturbation wavelength is comparable with the curvature scale; in this case the background pressure and energy density makes the Universe expand and we enter in the realm of cosmology. To study perturbations we consider a spatially flat FLRW perturbed solution in the conformal Newtonian gauge, namely,

$$
\begin{aligned}
d s^{2} & =a^{2} \eta_{\mu \nu} d x^{\mu} d x^{\nu}+2 a^{2}\left[\Psi(t, \vec{x}) d t^{2}+\Phi(t, \vec{x}) d \vec{x}^{2}\right] \\
& =a^{2}\left(\eta_{\mu \nu}+h_{\mu \nu}\right) d x^{\mu} d x^{\nu}
\end{aligned}
$$

while for the Stückelberg fields in the scalar sector we have

$\varphi^{0}=\phi(t)+\pi_{0}(t, \vec{x}), \quad \varphi^{a}=x^{a}+\partial_{a} \pi_{L}(t, \vec{x})$.

The mass paramaters $\lambda_{a}$ and $M_{a}$ in a FLRW background are similarly defined as in Eq. (3.4); it is convenient to introduce an overall factor $a^{4}{ }^{6}$ in the quadratic Lagrangian corresponding to the square root of minus the determinant of the background metric; namely,

$$
\begin{aligned}
L_{\text {mass }}^{(2)}= & \frac{M_{\mathrm{Pl}}^{2} a^{4}}{4}\left[\lambda_{0}^{2} h_{00}^{(u g) 2}+2 \lambda_{1}^{2} h_{0 i}^{(u g) 2}-2 \lambda_{4}^{2} h_{00}^{(u g)} h_{i i}^{(u g)}\right. \\
& \left.+\lambda_{3}^{2} h_{i i}^{(u g) 2}-\lambda_{2}^{2} h_{i j}^{(u g) 2}\right] .
\end{aligned}
$$

The expressions of $M_{a}$ are given in Appendix A. The conservation of the background EMT is equivalent to the equation of motion for $\bar{\varphi}^{0}=\phi$ :

$$
\rho^{\prime}=-3 \mathcal{H}(\rho+p) \Rightarrow \hat{M}_{0} \phi^{\prime \prime}=\left(\hat{M}_{0}+3 \hat{M}_{4}\right) \mathcal{H} \phi^{\prime}
$$

The quadratic Lagrangian for scalar perturbation in the Fourier basis can be unambiguously written as (see Appendix B)

\footnotetext{
${ }^{6}$ Notice that such an overall factor is not present in the definition given in Ref. [22].
} 


$$
\begin{aligned}
L_{s}^{\mathrm{FRW}} & =\frac{1}{2} Q^{\prime t} \cdot \boldsymbol{K} \cdot Q^{\prime}+\dot{Q}^{t} \cdot \boldsymbol{D} \cdot Q-\frac{1}{2} Q^{t} \cdot \boldsymbol{M} \cdot Q ; \\
\boldsymbol{K}^{t} & =\boldsymbol{K}, \quad \boldsymbol{M}^{t}=\boldsymbol{M}, \quad \boldsymbol{D}^{t}=-\boldsymbol{D},
\end{aligned}
$$

where $Q^{t}=\left(\Psi, \Phi, k^{2} \pi_{l}, k \pi_{0}\right)$ and ' denotes the derivative with respect of the conformal time. In our case we have that

$$
\begin{gathered}
\boldsymbol{K}=\left(\begin{array}{cccc}
0 & 0 & 0 & 0 \\
0 & -12 a^{2} M_{\mathrm{Pl}}^{2} & 0 & 0 \\
0 & 0 & a^{4} \frac{\left[\hat{M}_{1}+(p+\rho)\right]}{k^{2}} & 0 \\
0 & 0 & 0 & \frac{2 a^{4} \hat{M}_{0}}{k^{2} \phi^{2}}
\end{array}\right) ; \quad(4.6) \\
\boldsymbol{D}=M_{\mathrm{Pl}}^{2}\left(\begin{array}{cccc}
0 & 6 a^{2} \mathcal{H} & 0 & -\frac{a^{4} M_{0}}{k \phi^{\prime}} \\
-6 a^{2} \mathcal{H} & 0 & 0 & \frac{3 a^{4} M_{4}}{k \phi^{\prime}} \\
0 & 0 & 0 & -a^{4} \frac{\left(M_{1}-2 M_{4}\right)}{2 k \phi^{\prime}} \\
\frac{a^{4} M_{0}}{k \phi^{\prime}} & -\frac{3 a^{4} M_{4}}{k \phi^{\prime}} & a^{4} \frac{\left(M_{1}-2 M_{4}\right)}{2 k \phi^{\prime}} & 0
\end{array}\right) .
\end{gathered}
$$

We denoted by $\mathcal{H}=a^{\prime} / a$ the Hubble parameter in conformal time. The mass matrix $\boldsymbol{M}$ is not very illuminating. Differently from the analysis involving the Stückelberg fields in flat space, this time the effect of the background on the FLRW metric is consistently taken into account and

$$
\mathcal{H}^{2}=\frac{a^{2} \rho}{6 M_{\mathrm{Pl}}^{2}}, \quad \mathcal{H}^{\prime}=-\frac{a^{2}(\rho+3 p)}{12 M_{\mathrm{Pl}}^{2}} .
$$

The field $\Psi$ has an algebraic equation of motion and can be integrated out; the resulting Lagrangian, after suitable integration by parts, can be again put in the form of Eq. (4.5). As a final step, a second field can be integrated out. Indeed, defining

$$
q=\Phi-\frac{\mathcal{H}}{\phi^{\prime}} \pi_{0},
$$

when the quadratic action is expressed in terms of $q, \pi_{l}$, and $\pi_{0}$, it turns out that $\pi_{0}$ can be integrated out. The action has the very same general form (4.5) but with $2 \times 2$ matrices:

$$
\begin{gathered}
\boldsymbol{K}=\frac{M_{\mathrm{Pl}}^{2}}{f}\left(\begin{array}{cc}
12 a^{2} \hat{M}_{0}\left(3 a^{2} \mathcal{M}_{1}+4 M_{\mathrm{Pl}}^{2} k^{2}\right) & 12 a^{4} \hat{M}_{0} \mathcal{M}_{1} \\
12 a^{4} \hat{M}_{0} \mathcal{M}_{1} & 4 \rho a^{4} \mathcal{M}_{1}
\end{array}\right), \\
\mathcal{M}_{1}=\hat{M}_{1}+\rho+p, \quad f=4 M_{\mathrm{Pl}}^{2} k^{2} \rho-3 a^{2} \mathcal{M}_{1}\left(\rho-\hat{M}_{0}\right),
\end{gathered}
$$

and

$$
\boldsymbol{D}=12 a^{2} k^{2} M_{\mathrm{Pl}}^{4}\left(\begin{array}{cc}
0 & -\frac{\left(\hat{M}_{1}-2 \hat{M}_{4}\right) \mathcal{H}}{f} \\
\frac{\left(\hat{M}_{1}-2 \hat{M}_{4}\right) \mathcal{H}}{f} & 0
\end{array}\right) .
$$

The expression of $\boldsymbol{M}$ is not particularly illuminating and will not be given here. What is important is to find the condition for which the total energy is positive definite in the large $k$ limit, namely, when $k^{2} \gg \mathcal{H}^{2}$, to confirm the pure Stückelberg analysis in flat space. Proceeding as for the case of flat space, in the large $k$ limit, we find the kinetic energy is positive if

$$
M_{0}>0 \quad \text { and } \quad \hat{M}_{1}+(p+\rho)>0 .
$$

Moreover, on can easily see that such a condition, together with

$$
\hat{M}_{0}<\rho,
$$

enforce that the kinetic energy is positive definite for any $k$. Turning to the mass matrix in the energy, it is positive if

$$
M_{2}>M_{3} \text { and } \quad M_{1}<0 .
$$

Finally, looking at vector modes it is easy to see that [22] stability requires

$$
\hat{M}_{1}+(p+\rho)>0 \text { and } \quad M_{2}>0 .
$$

As a result, the stability conditions are the same as the ones derived with the previous Stückelberg analysis; in addition when $\hat{M}_{0}<\rho$, ghost instability is forbidden at any $k$. The only instability found is a Jeans instability when, for $k<k_{J}$, one of the eigenvalues of the mass matrix of the total energy becomes negative. Such gravitational instability is typical of any self-gravitating system.

As a technical comment, we note that taking the limit $\mathcal{H} \rightarrow 0$, via the background equations, one is led again to $p=\rho=0$ and we are back to the well-known problems discussed in the previous section.

Let us consider a number of concrete examples.

(i) Perfect fluid

This is the simplest case one can imagine. Flat space stability actually coincides with thermodynamical stability [25]. From the fluid Lagrangian of the form $U(b, Y)$, we have that $M_{1}=M_{2}=0$. The transverse spin 1 mode has a degenerate dispersion relation related to the classical conservation of vorticity in a perfect fluid and the dynamics of tensor modes is not modified. Stability conditions are simpler and come only from Eq. (2.7) with $M_{1}=0$ and are equivalently from the corresponding expression in FLRW. Thus, 


$$
M_{0}=\frac{\phi^{2} U_{Y Y}}{2 a^{2} M_{\mathrm{Pl}}^{2}}>0, \quad p+\rho>0,
$$

which can be easily satisfied. The only exception is when the null energy condition is violated.

(ii) Superfluid

A superfluid is the simplest example of a medium with $M_{1} \neq 0$. Being still a fluid, $M_{2}=0$. The Lagrangian has the general form $U(b, Y, X)$, see Appendix A and Ref. [22] for the definition of the relevant operators. Clearly the most stringent stability conditions are the ones involving $M_{1}$ that for a superfluid is given, together with the background value of $X$ on FLRW, by

$$
M_{1}=\frac{2 \phi^{\prime 2} U_{X}}{M_{\mathrm{Pl}}^{2} a^{2}}, \quad X=-\frac{\phi^{2}}{a^{2}} .
$$

Moreover, for superfluids [22], see Appendix A, we have that

$$
p+\rho=\frac{\phi^{\prime} U_{Y}}{a}-\frac{2 \phi^{\prime 2} U_{X}}{a^{2}}-\frac{U_{b}}{a^{3}} .
$$

Then the stability condition involving $M_{1}$ reads simply

$$
U_{X}<0, \quad \frac{\phi^{\prime} U_{Y}}{a}-\frac{U_{b}}{a^{3}}>0 ;
$$

remarkably, there is a perfect cancellation of $U_{X}$ between $M_{1}$ and $\rho+p$ rendering such condition easy to satisfy. Notice that $a^{-1} \phi^{\prime} U_{Y}-a^{-3} U_{b}$ is just $p+\rho$ for the normal component of the superfluid; see Appendix A.

(iii) Supersolid

Take a supersolid (not the most general one) described by the Lagrangian $U\left(b, Y, X, \tau_{n}\right)$ with $n=1,2,3$. The presence of $\tau_{n}$ turns on $M_{2}$; however, the cancellation of $U_{X}$ in the conditions involving $M_{1}$ still takes place and we have that

$$
\begin{aligned}
& U_{X}<0, \\
& \frac{\phi^{\prime} U_{Y}}{a}-\frac{U_{b}}{a^{3}}-2\left(\frac{U_{\tau_{1}}}{a^{3}}+\frac{2 U_{\tau_{2}}}{a^{4}}+\frac{3 U_{\tau_{1}}}{a^{3}}\right)>0,
\end{aligned}
$$

which again is not very restrictive. The condition is just $\rho+p$ for a finite temperature solid; thus, as for superfluids, the effect of $M_{1}$ is to cancel the contribution from the superfluid component; see Appendix A.

(iv) Most general supersolid

Consider now the case of the most general solid whose Lagrangian $U$ contains all ten operators, rotational invariant operators; see Appendix A.
While terms of the form $U_{y_{m}}, m=0,1,2,3$, contribute to $M_{1}$, there is no contribution in $p+$ $\rho$ being of the form $y_{m} U_{y_{m}}$ and $y_{m}=0$ at the background level. As a result the stability conditions can be rather restrictive for the most general supersolid.

The bottom line is that the stability conditions are easily satisfied with the possible exception of the most general supersolid, where the restriction on $M_{1}$ is nontrivial. It is also worthwhile to stress that we have studied the stability conditions in the worse case where the medium itself is dominating the Universe and thus the scale of $\rho$ and $p$ are the same as $\hat{M}_{a}$. For instance, when radiation is dominant we expect that very similar stability conditions should hold with $p$ and $\rho$ associated with the dominant component (ultrarelativistic particles) and $\hat{M}_{a} \ll p+\rho$, making stability not an issue.

\section{MASSIVE GRAVITY PHASES}

Here we briefly describe what happens once the selfgravitating nature of the medium has been taken into account. The special case of a de Sitter background, where $p+\rho=0$, which incidentally is naturally the one selected by the Lorentz invariant (LI) quadratic Lagrangians, will be analyzed in a forthcoming paper; here we just report, for completeness, the main results.

(1) The most interesting and new case is when basically no tuning on $U$ is required and this also applies to the resulting masses. ${ }^{7}$ The vacuum state breaks Lorentz invariance, which in turn allows the existence of nontrivial pressure and energy density background; stability can be achieved when $p+\rho \neq 0$. The full six d.o.f. of the theory can be stable at quadratic order.

There are two critical points in the parameter space where the kinetic matrix is degenerate and the above analysis has to be repeated (we always require $p, \rho \neq 0)$

(2) When $\hat{M}_{0}=M_{\mathrm{Pl}}^{2} \lambda_{0}^{2}-\rho=0$ we have at least one d.o.f. less in the scalar sector, for a total of 5 d.o.f. The conservation of the background EMT is equivalent to (4.4) and for $\hat{M}_{0}=0$ (with $\mathcal{H} \neq 0$ ) it implies that also $\hat{M}_{4}=M_{\mathrm{Pl}}^{2} \lambda_{4}^{2}-p / 2=0$. No constraint on $\hat{M}_{4}$ exists on a Minkowski space background where $\mathcal{H}=0$.

(a) In the Lorentz breaking (LB) case we have only one propagating scalar whose kinetic term is proportional to $k^{2} \mathcal{H}^{2}(1+w)$ and mass to $\left(M_{2}-M_{3}\right)$ (in the large $k$ limit). Thus, when $w=-1$ the kinetic term for such a mode vanishes.

\footnotetext{
${ }^{7}$ In particular we have to avoid the zeros in the kinetic matrix (4.10), $\mathcal{M}_{1}, M_{0} \neq 0$.
} 
(b) For a LI quadratic Lagrangian and with $\rho+p=0$, the requirement of $M_{4}=0$, for the existence of an FLRW background, gives

$$
\left\{\begin{array}{l}
M_{0}=A+B-\frac{\rho}{2}=0 \\
M_{3,4}=B+\frac{\rho}{2}=0
\end{array} \Rightarrow M_{1,2}=-A+\rho=0 .\right.
$$

As a result, a LI self gravitating medium in such a phase has all masses $M_{i}$ zero and the parameters $A$ and $B$ are fine tuned in accord with $M_{\mathrm{Pl}}^{2} A=\rho, M_{\mathrm{Pl}}^{2} B=-\rho / 2$, and no scalar and vector propagates at the quadratic order. Only two massless tensor modes are present. In Minkowski space, keeping $M_{4} \neq 0$ also $M_{1} \neq 0$ and then tensors and transverse vectors propagate and we get 5 d.o.f.. In the contest of the decoupling limit of Pauli-Fierz massive gravity, the vanishing of the kinetic term in the scalar sector is dealt by changing the Stückelberg content by adding an additional scalar [27,2].

(3) When $\hat{M}_{1}+\rho+p=M_{\mathrm{Pl}}^{2} \lambda_{1}^{2}+\rho=0$, only a single scalar and two tensor modes propagate for a total of $\leq 3$ d.o.f.; such a phase can be implemented in a nonperturbative way and can be protected by a symmetry $[1,5]$.

(a) In the LB theory the kinetic term for the only propagating scalar mode is given by

$$
K=\frac{1}{2 \mathcal{H}^{2}}\left[a^{4} \hat{M}_{0}+\frac{\left(\hat{M}_{4} a^{2}+3(1+w) \mathcal{H}^{2}\right)^{2}}{\hat{M}_{2}-\hat{M}_{3}}\right],
$$

which is positive defined for $M_{0}>0, M_{2}>M_{3}$. (b) In the LI case being $\lambda_{1}^{2}=-A$ implies $A=\rho$ and, being $p+\rho=0$, we have

$$
M_{1,2}=0, \quad \hat{M}_{0,3,4}=B+\frac{\rho}{2} .
$$

From Eq. (5.2) we get $K=0$ and then no scalar mode propagates. Likewise in GR, only massless tensor modes are present.

Let us comment on the Higuchi bound $[28,29]$. Such a bound on the Pauli-Fierz mass in dS spacetime is derived when the massive spin 2 action gives no contribution to the background [30,31]; namely, no linear term is present. In the dRGT massive gravity model, a dS background exists when a non-flat time-dependent reference metric [32] (basically the same of the one found in the contest of bigravity [33,34]) is considered; in this case the Eguchi bound is recovered. In our approach, by definition, the medium is self-gravitating and the above considerations do not apply.

\section{CONCLUSIONS}

We have analyzed the low energy phononlike excitations of generic media (fluids, superfluids, solids, and supersolids) on Minkowski space showing that typically no instability is present. It turns out that when dynamical gravity is added to the game, in the unitary gauge, self-gravitating media are equivalent to massive gravity and six d.o.f. are present. On the other hand, massive gravity theories with six d.o.f. on Minkowski space are plagued by ghost instabilities $[4,5,1-3]$ and a great effort has been devoted trying to find a nonperturbative way to project out the unwanted (ghostlike) sixth mode. While giving up Lorentz symmetry helps to get rid of the vDVZ discontinuity [6], generic massive gravity theory still propagates six d.o.f. $[5,8,9]$. The standard route is to devise a special form of the massive gravity Lagrangian in oder to get five or less d.o.f. [1,7-9]; with or without Lorentz invariance, at the linearized level this corresponds to set $\lambda_{0}=0$ in Eq. (3.4). We reconcile the two apparent contradictory facts by taking into account the pivotal role played by the pressure and energy density of the medium. The point is that flat space is not a consistent background for a selfgravitating medium and for massive gravity, unless the background pressure and energy density is set to zero. In such case the generic stability conditions for a medium are badly violated. This is not surprising — when $p=\rho=0$ the medium picture breaks down simply because there is no medium. To consistently study the dynamics of a selfgravitating media in the presence of dynamical gravity one has to change the background, taking a FLRW universe; the stability analysis around such background again shows that generically no ghost instability is present and actually for modes with wavelength much smaller than the curvature scale $\mathcal{H}^{-1}$ the flat space results with gravity switched off are recovered. Interestingly, the fluid picture is incompatible with the requirement of Lorentz invariance of the medium energymomentum tensor and leads again to $p=\rho=0$ and inevitably the Lagrangian has to be tuned to get less than six d.o.f. As a result, the spontaneous breaking of Lorentz invariance is instrumental to open up the stability window for a massive gravity-self-gravitating medium with six d.o.f. Such a breaking is an emergent low-energy phenomenon triggered by the nonvanishing homogenous background value of the Stïckelberg scalar fields due to the presence of a medium with nonzero energy density and pressure. One of the main theoretical and phenomenological features of such a model is that the Einstein-Hilbert part of the action is untouched, keeping the dynamics of the spin two graviton very close to GR, except for a massive deformed dispersion relation

$$
\omega=\sqrt{k^{2}+m^{2}} \simeq k+\frac{m^{2}}{2 k}+\cdots, \quad k^{2} \gg m^{2} .
$$

For a typical frequency of the order some Hertz, observations $[14,15]$ require that the graviton velocity is very close to $c=1$ : 


$$
\Delta v \sim \frac{m^{2}}{k^{2}} \leq 10^{-15} \Rightarrow m<10^{-22} \mathrm{eV}
$$

Such a bound on the mass is not as stringent as requiring that the mass scale is of the order of the present Hubble parameter $\sim 10^{-33} \mathrm{eV}$ if massive gravity is relevant for dark energy.

\section{APPENDIX A: SELF GRAVITATING MEDIA}

The operators entering the Lagrangian describing the low energy dynamics of a general isotropic medium minimally coupled with gravity can be given in terms of matrix $[21,22]$

$$
C^{A B}=g^{\mu \nu} \partial_{\mu} \varphi^{A} \partial_{\nu} \varphi^{B}, \quad A, B=0,1,2,3
$$

by

$$
\begin{aligned}
X & =C^{00}, \quad \tau_{a}=\operatorname{Tr}\left(\boldsymbol{B}^{a}\right) \quad a=1,2,3, \\
y_{n} & =\operatorname{Tr}\left(\boldsymbol{B}^{n} \cdot \boldsymbol{Z}\right) \quad n=0,1,2,3 ; \\
(\boldsymbol{B})^{a b} & =C^{a b} \equiv B^{a b}, \quad(\boldsymbol{Z})^{a b}=C^{0 b} C^{0 b} .
\end{aligned}
$$

For a perfect fluid $U$ can be taken as a function of only two operators $b$ and $Y$ defined by

$$
\begin{aligned}
b & =\sqrt{\operatorname{det}\left(B^{a c}\right)}, a, c=1,2,3, \\
u^{\mu} & =\frac{\epsilon^{\mu \alpha \beta \gamma} \partial_{\alpha} \varphi^{1} \partial_{\beta} \varphi^{2} \partial_{\gamma} \varphi^{3}}{b \sqrt{g}}, \quad Y=u^{\mu} \nabla \varphi^{0} .
\end{aligned}
$$

The 4-vector $u^{\mu}$ is the fluid's velocity and $u^{\mu} \partial_{\mu} \varphi^{a}=0$; thus, $\varphi^{a}$ can be interpreted as the spatial Lagrangian (comoving) coordinates of the fluid, while $\varphi^{0}$ represents the fluid's clock. Together with $b$, and $Y, X,\left\{\tau_{n}\right\}$ and $\left\{y_{m}\right\}$ form a set of ten independent rotational invariant operators, thus the Lagrangian of a general medium will have the form $U\left(b, Y, X, \tau_{a}, y_{n}\right)$. The pressure and the energy density are given by [22]

$$
\begin{gathered}
\rho=-U+Y U_{Y}-2 Y^{2} U_{X} \\
p=U-b U_{b}-\frac{2}{3} \sum_{n=1}^{3} n \tau_{n} U_{\tau_{n}}-\frac{2}{3} \sum_{n=0}^{3} n y_{n} U_{y_{n}} \\
-\frac{2}{3} U_{X}\left(Y^{2}+X\right) .
\end{gathered}
$$

In the case of a perfect fluid we simply have that $\rho=$ $-U+Y U_{Y}$ and $p=U-b U_{b}$.

$$
\rho=-U+Y U_{Y}, \quad p=U-b U_{b} .
$$

The mass parameters $\left\{M_{a}\right\}$ are defined separating the contribution from $\left\{\lambda_{a}\right\}$ the part that is related to the background pressure and energy density, this is done by Eq. (3.5) which leads to

$$
\begin{aligned}
M_{0}= & \frac{\phi^{\prime 2}}{2 a^{4} M_{\mathrm{Pl}}^{2}}\left[a^{2}\left(U_{Y Y}-2 U_{X}\right)-4 a \phi^{\prime} U_{Y X}+4 \phi^{\prime 2} U_{X X}\right] ; \\
M_{1}= & \frac{2 \phi^{\prime 2}}{M_{\mathrm{Pl}}^{2}}\left[a^{-2} U_{X}+\sum_{n=0}^{3} a^{-4-2 n} U_{y_{n}}\right] ; \\
M_{2}= & -\frac{2}{M_{\mathrm{Pl}}^{2}} \sum_{m=1}^{3} n^{2} U_{\tau_{n}} ; \\
M_{3}= & \frac{1}{M_{\mathrm{Pl}}^{2}}\left(2 \sum_{m, n=1}^{3} m n a^{-2 m-2 n} U_{\tau_{m} \tau_{n}}+2 \sum_{n} n a^{-3-2 n} U_{b \tau_{n}}\right. \\
& \left.-\sum_{n, m=1}^{3} a^{-2 n} U_{\tau_{n}}+\frac{1}{2 a^{6}} U_{b^{2}}\right) ; \\
M_{4}= & \frac{1}{M_{\mathrm{Pl}}^{2}}\left[\frac{\phi^{\prime}}{2}\left(2 \sum_{m, n=1}^{3} a^{1-2 n} U_{Y \tau_{n}}-\frac{U_{Y}}{a}+\frac{U_{b Y}}{a^{4}}\right)\right. \\
& \left.+\phi^{\prime 2}\left(-2 \sum_{n=1}^{3} a^{-2-2 n} U_{X \tau_{n}}+\frac{U_{X}}{a^{2}}-\frac{U_{b X}}{a^{5}}\right)\right] .
\end{aligned}
$$

In Minkowski space the mass parameters are obtained from the above expression by setting $a=\phi^{\prime}=1$.

\section{APPENDIX B: UNITARY VS NEWTONIAN GUAGE IN FRW SPACE}

The quadratic action for scalar perturbations in a Fourier basis can be unambiguously written in matrix notation as

$$
\mathcal{L}_{2}=\frac{1}{2} Q^{\prime t} \cdot \boldsymbol{K} \cdot Q^{\prime}+Q^{\prime t} \cdot \boldsymbol{D} \cdot Q-\frac{1}{2} Q^{t} \cdot \boldsymbol{M} \cdot Q
$$

the relevant metric and scalar field perturbations are collected in $Q$, a vector with 4 components. Then we have the $4 \times 4$ matrices: ${ }^{8}$ The matrix $\boldsymbol{K}$ is the kinetic matrix and its symmetric by construction as the mass matrix $\boldsymbol{M}$. If a nondynamical field is present, it can be integrated out and the result will be still of the form (B1) with a new $Q$ and $\boldsymbol{K}$, $\boldsymbol{D}$, and $\boldsymbol{M}$ of lower dimension. In order to study the stability of the system described by (B1) we simply impose the positivity of the Hamiltonian. The conjugate momenta are

$$
\Pi=\frac{\partial \mathcal{L}_{2}}{\partial Q^{\prime t}}=\boldsymbol{K} \cdot Q^{\prime}+\boldsymbol{D} \cdot Q
$$

When $\boldsymbol{K}$ is nondegenerate we are sure that no constraint is present. The total energy $E$, basically the Hamiltonian, expressed as a quadratic form in $Q^{\prime}$ and $Q$ is

\footnotetext{
${ }^{8}$ If the matrix $D$ in Eq. (B1) is not antisymmetric, it is possible by an integration by parts to kill its symmetric part; as a consequence, in Eq. (B1) one has to perform the following replacement: $\boldsymbol{D} \rightarrow \frac{\boldsymbol{D}-\boldsymbol{D}^{t}}{2}$ and $\boldsymbol{M} \rightarrow \boldsymbol{M}+\frac{\boldsymbol{D}^{\prime}+\boldsymbol{D}^{\prime t}}{2}$.
} 


$$
\begin{aligned}
E & =Q^{\prime t} \cdot\left(\boldsymbol{K} \cdot Q^{\prime}+\boldsymbol{D} \cdot Q\right)-\mathcal{L}_{2} \\
& =\frac{1}{2}\left(Q^{\prime t} \cdot \boldsymbol{K} \cdot Q^{\prime}+Q^{t} \cdot \boldsymbol{M} \cdot Q\right) .
\end{aligned}
$$

Equivalently, the Hamiltonian in terms of $\Pi$ and $Q$ can be written as

$H=\frac{1}{2}\left[\left(\Pi^{t}-Q^{t} \cdot \boldsymbol{D}^{t}\right) \cdot \boldsymbol{K}^{-1} \cdot(\Pi-\boldsymbol{D} \cdot Q)+Q^{t} \cdot \boldsymbol{M} \cdot Q\right]$.

It is then clear that the positivity of the Hamiltonian or the energy is equivalent to the positivity of the kinetic matrix $\boldsymbol{K}$ and mass matrix $\boldsymbol{M}$, independently from $\boldsymbol{D}$.

In the unitary gauge all scalar perturbations in the metric according with

$d s^{2}=a^{2}\left(\eta_{\mu \nu}+h_{\mu \nu}^{(U)}\right) d x^{\mu} d x^{\nu}, \quad h_{00}^{(U)}=2 \Psi$,

$h_{0 i}^{(U)}=\partial_{i} v, \quad h_{i j}^{(U)}=2 \Phi \delta_{i j}+\partial_{i} \partial_{j} \sigma$,

while the scalars fields are frozen in their background value

$$
\varphi^{0}=\phi(t), \quad \varphi^{a}=x^{a} \quad a=1,2,3 .
$$

The relevant fields are $Q^{(U)}=(\Psi, \Phi, v, \sigma)$ and from the expansion of Eq. (3.1) we get

$$
\begin{aligned}
& \boldsymbol{K}^{(U)}=M_{\mathrm{Pl}}^{2}\left(\begin{array}{cccc}
0 & 0 & 0 & 0 \\
0 & -12 & 0 & 2 k^{2} \\
0 & 0 & 0 & 0 \\
0 & 2 k^{2} & 0 & 0
\end{array}\right), \\
& \boldsymbol{D}^{(U)}=M_{\mathrm{Pl}}^{2}\left(\begin{array}{cccc}
0 & 0 & 0 & 0 \\
0 & 0 & -2 k^{2} & 0 \\
0 & 2 k^{2} & 0 & 0 \\
0 & 0 & 0 & 0
\end{array}\right) ;
\end{aligned}
$$

while $\boldsymbol{M}^{(U)}$ will be not given here. Notice the in the unitary gauge in the quadratic Lagrangian nor $p$ neither $\rho$ is present, thus imposing the background equation of motions $p=\rho=0$ is immaterial. From the form of $K$ it is clear that both $\Psi$ and $v$ are auxiliary fields and can be integrated out when $\lambda_{0} \neq 0$ and $\lambda_{1} \neq 0$. After integrating out those fields we arrive at an effective quadratic Lagrangian still of the form of Eq. (4.5) with $Q=(\Phi, \sigma)$ with

$\boldsymbol{K}_{2}^{U}=M_{\mathrm{Pl}}^{2}\left(\begin{array}{cc}-4\left(4 k^{2}+3 \lambda_{1}\right) / \lambda_{1} & 2 k^{2} \\ 2 k^{2} & 0\end{array}\right), \quad \boldsymbol{D}_{2}^{U}=0$

The expression of the mass matrix is not relevant for us. Clearly, there are two propagating d.o.f. and one is a ghost.

In the Newtonian gauge the scalar perturbations are both in the metric and in the scalar fields, namely,

$$
\begin{aligned}
\varphi^{0} & =\phi(t)+\pi_{0}, \quad \varphi^{a}=x^{a}+\partial_{a} \pi_{l}, \\
h_{\mu \nu}^{(N)} & =2 \Psi \delta_{\mu}^{0} \delta_{\nu}^{0}+2 \Phi \delta_{\mu}^{i} \delta_{\nu}^{i},
\end{aligned}
$$

and in the Newtonian gauge $Q^{(N)}=\left(\Psi, \Phi, k^{2} \pi_{l}, k \pi_{0}\right)$ and

$$
\boldsymbol{K}^{(N)}=\left(\begin{array}{cccc}
0 & 0 & 0 & 0 \\
0 & -12 M_{\mathrm{Pl}}^{2} & 0 & 0 \\
0 & 0 & 2 M_{\mathrm{Pl}}^{2} \lambda_{0}-\rho & 0 \\
0 & 0 & 0 & k^{2}\left(M_{\mathrm{Pl}}^{2} \lambda_{1}+\rho\right) ;
\end{array}\right)
$$

the expression for $\boldsymbol{D}^{(N)}$ and $\boldsymbol{M}^{(N)}$ will be omitted. Notice that this time the quadratic Lagrangian depends on $p$ and $\rho$ if the background equations are not imposed. Now $\boldsymbol{K}$ is rank three and there are three d.o.f. in sharp contrast with the unitary gauge computation. The only way to recover the same result if to impose $p=\rho=0$; then also $\pi_{0}$ can be integrated out and the we end up with two d.o.f. and one is a ghost.
[1] V. A. Rubakov and P. G. Tinyakov, Infrared-modified gravities and massive gravitons, Phys. Usp. 51, 759 (2008).

[2] K. Hinterbichler, Theoretical aspects of massive gravity, Rev. Mod. Phys. 84, 671 (2012).

[3] C. de Rham, Massive gravity, Living Rev. Relativity 17, 7 (2014).

[4] D. G. Boulware and S. Deser, Can gravitation have a finite range?, Phys. Rev. D 6, 3368 (1972).

[5] S. L. Dubovsky, Phases of massive gravity, J. High Energy Phys. 10 (2004) 076.
[6] V. Rubakov, Lorentz-violating graviton masses: Getting around ghosts, low strong coupling scale and VDVZ discontinuity, arXiv:hep-th/0407104.

[7] C. de Rham, G. Gabadadze, and A. J. Tolley, Resummation of Massive Gravity, Phys. Rev. Lett. 106, 231101 (2011).

[8] D. Comelli, F. Nesti, and L. Pilo, Massive gravity: A general analysis, J. High Energy Phys. 07 (2013) 161.

[9] D. Comelli, F. Nesti, and L. Pilo, Nonderivative modified gravity: A classification, J. Cosmol. Astropart. Phys. 11 (2014) 018. 
[10] H. van Dam and M. J. G. Veltman, Massive and massless Yang-Mills and gravitational fields, Nucl. Phys. B22, 397 (1970).

[11] V. I. Zakharov, Linearized gravitation theory and the graviton mass, JETP Lett. 12, 312 (1970).

[12] A. I. Vainshtein, To the problem of nonvanishing gravitation mass, Phys. Lett. B 39, 393 (1972).

[13] B. Bellazzini, F. Riva, J. Serra, and F. Sgarlata, Beyond Amplitudes' Positivity and the Fate of Massive Gravity, Phys. Rev. Lett. 120, 161101 (2018).

[14] B. P. Abbott et al. (Virgo, LIGO Scientific Collaborations), GW170817: Observation of Gravitational Waves from a Binary Neutron Star Inspiral, Phys. Rev. Lett. 119, 161101 (2017).

[15] B. P. Abbott et al. (Virgo, Fermi-GBM, INTEGRAL, LIGO Scientific Collaborations), Gravitational waves and gamma rays from a binary neutron star merger: GW170817 and GRB 170817A, Astrophys. J. 848, L13 (2017).

[16] P. Creminelli and F. Vernizzi, Dark Energy after GW170817, Phys. Rev. Lett. 119, 251302 (2017).

[17] J. Sakstein and B. Jain, Implications of the Neutron Star Merger GW170817 for Cosmological Scalar-Tensor Theories, Phys. Rev. Lett. 119, 251303 (2017).

[18] J. M. Ezquiaga and M. Zumalacárregui, Dark Energy after GW170817: Dead Ends and the Road Ahead, Phys. Rev. Lett. 119, 251304 (2017).

[19] T. Baker, E. Bellini, P. G. Ferreira, M. Lagos, J. Noller, and I. Sawicki, Strong Constraints on Cosmological Gravity from GW170817 and GRB 170817A, Phys. Rev. Lett. 119, 251301 (2017).

[20] S. Dubovsky, T. Gregoire, A. Nicolis, and R. Rattazzi, Null energy condition and superluminal propagation, J. High Energy Phys. 03 (2006) 025.

[21] G. Ballesteros, D. Comelli, and L. Pilo, Massive and modified gravity as self-gravitating media, Phys. Rev. D 94, 124023 (2016).
[22] M. Celoria, D. Comelli, and L. Pilo, Fluids, superfluids and supersolids: Dynamics and cosmology of self gravitating media, J. Cosmol. Astropart. Phys. 09 (2017) 036.

[23] S. Dubovsky, L. Hui, A. Nicolis, and D. T. Son, Effective field theory for hydrodynamics: thermodynamics, and the derivative expansion, Phys. Rev. D 85, 085029 (2012).

[24] G. Ballesteros and B. Bellazzini, Effective perfect fluids in cosmology, J. Cosmol. Astropart. Phys. 04 (2013) 001.

[25] G. Ballesteros, D. Comelli, and L. Pilo, Thermodynamics of perfect fluids from scalar field theory, Phys. Rev. D 94, 025034 (2016).

[26] D. Blas, D. Comelli, F. Nesti, and L. Pilo, Lorentz breaking massive gravity in curved space, Phys. Rev. D 80, 044025 (2009).

[27] N. Arkani-Hamed, H. Georgi, and M. D. Schwartz, Effective field theory for massive gravitons and gravity in theory space, Ann. Phys. (Amsterdam) 305, 96 (2003).

[28] A. Higuchi, Forbidden mass range for spin-2 field theory in de Sitter space-time, Nucl. Phys. B282, 397 (1987).

[29] A. Higuchi, Symmetric tensor spherical harmonics on the $N$ sphere and their application to the de Sitter group $\mathrm{SO}(N, 1)$, J. Math. Phys. 28, 1553 (1987).

[30] S. Deser and A. Waldron, Stability of massive cosmological gravitons, Phys. Lett. B 508, 347 (2001).

[31] L. Grisa and L. Sorbo, Pauli-Fierz gravitons on FriedmannRobertson-Walker background, Phys. Lett. B 686, 273 (2010).

[32] M. Fasiello and A. J. Tolley, Cosmological perturbations in massive gravity and the Higuchi bound, J. Cosmol. Astropart. Phys. 11 (2012) 035.

[33] D. Comelli, M. Crisostomi, F. Nesti, and L. Pilo, FRW Cosmology in ghost free massive gravity, J. High Energy Phys. 03 (2012) 067.

[34] D. Comelli, M. Crisostomi, and L. Pilo, Perturbations in massive gravity cosmology, J. High Energy Phys. 06 (2012) 085 . 